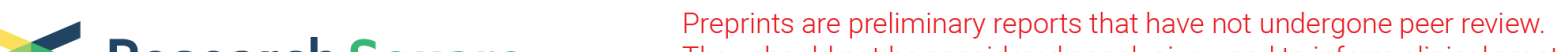 Research Square
or referenced by the media as validated information.
}

\section{Comparison of In-hospital Mortality Prognostic Accuracy of the pSOFA Score and SIRS Criteria Among Children With Infection Admitted to Pediatric Intensive Care Units: A Retrospective Multi-center Cohort Study}

\section{Chun Zhao}

Shandong Provincial Hospital Affiliated to Shandong First Medical University https://orcid.org/00000002-5405-9177

\section{Meiyun Xin}

Affiliated Hospital of Jining Medical University

Jing Li

Qingdao Women and Children's Hospital

Jinfang Zhao

Shandong Provincial Hospital Affiliated to Shandong First Medical University

\section{Yujuan Wang}

Shandong Provincial Hospital Affiliated to Shandong First Medical University

\section{Wei Wang}

Shandong Provincial Hospital Affiliated to Shandong First Medical University

\section{Qian Gao}

Shandong Provincial Hospital,Cheeloo College of Medicine,Shandong University

Jie Chen

Shandong Provincial Hospital,Cheeloo College of Medicine,Shandong University

\section{Qiwei Wang}

Shandong Provincial Hospital Affiliated to Shandong First Medical University

\section{Youpeng Jin ( $\nabla$ jinyp79@126.com )}

Shandong Provincial Hospital Affiliated to Shandong First Medical University https://orcid.org/00000002-6357-2810

\section{Research}

Keywords: simple-pSOFA, pSOFA, Children, Critical care, Mortality, Infection, Sepsis, SIRS 
DOl: https://doi.org/10.21203/rs.3.rs-465406/v1

License: (c) (1) This work is licensed under a Creative Commons Attribution 4.0 International License. Read Full License 


\section{Abstract}

Background: The sepsis version 3.0 highlights the co-existence of infection with organ dysfunction, assessed by the Sequential Organ Failure Assessment (SOFA) score. However, the prognostic accuracy of the Pediatric SOFA score (pSOFA) for in-hospital mortality was not fully validated. We compared the performance of the PSOFA with the SIRS criteria as predictors of in-hospital mortality in children with infectious disease and evaluate whether the pSOFA is suitable to be used in pediatric intensive care units (PICU) in Shandong Province, China.

Methods::A retrospective multicenter cohort study was conducted on children hospitalized with confirmed or suspected infection, between January 2011 and December 2019, in three PICUs of three tertiary care hospitals in Shandong Province. The maximum pSOFA score and SIRS criteria within the first $24 \mathrm{hr}$ of PICU admission were calculated. Univariate and multivariate logistic regressions were conducted to analyze the risk factors for in-hospital mortality. Three sub-components of the pSOFA score with relatively good predictive value were identified according to the Yuden Index and formed a simple-pSOFA score. The efficiency and diagnostic ability of these scores were compared by measuring the area under the receiver operating characteristic curve (AUROC) and Delong's test.

Results: 1092 patients were included and the overall in-hospital mortality was $23.4 \%$. Multivariate logistic regressions showed that high $24 \mathrm{~h}$ pSOFA score, bloodstream as the main infection site, and the need for MV were the risk factors for in-hospital mortality. The AUROC analysis verified that simple-pSOFA had the best diagnostic ability in predicting in-hospital mortality of infected children compared with the $24 \mathrm{~h}$ pSOFA score and SIRS criteria. The sensitivity and specificity of $24 \mathrm{~h}$ pSOFA score $\geq 2$ were the highest, $97.6 \%$ and $78.9 \%$, respectively.

Conclusions: The pSOFA score was superior to SIRS criteria in identifying children with infection and a high risk of mortality, but it is expensive and time-consuming to complete the pSOFA score. SimplepSOFA score is superior to PSOFA score in early identification of infected children with a high risk of death, but further validation studies with a larger size is needed.

\section{Background}

Infectious diseases are common in children, and severe cases could induce sepsis, multiple organ failure, and even death. In recent years, the incidence of sepsis in children has increased [1, 2]. Of the 7.6 million deaths in children age 5 or younger in $2010,64.0 \%$ (4.879 million) were attributable to sepsis or sepsis shock caused by a severe infectious disease $[3,4]$. Even if a child survived, their quality of life was diminished $[5,6]$. A European study found that although 24 percent of children had no previous disease history, 31 percent of children who survived sepsis developed disabilities [7]. In addition, a SPROUT study showed that 17 percent of children surviving sepsis had at least one disability [8]. Therefore, early and accurate diagnosis of sepsis is key to improving the prognosis of infected children and arranging timely and reasonable follow-up treatment. 
The old definitions of sepsis, versions 1.0 and 2.0, used SIRS criteria $[9,10]$, but its value at predicting the severity of patient illness has been questioned $[11,12]$. The definition of sepsis 3.0, introduced in 2016, highlights the co-existence of infection with organ dysfunction, which is assessed using the Sequential Organ Failure Assessment (SOFA) score. The sepsis 3.0 task force validated the SOFA score's ability to diagnose adult patients with suspected and confirmed infections [13]. Therefore, Sepsis version 3.0 is expected to be widely adopted and the SOFA score is now used to give a prognosis to patients with confirmed or suspected infections. However, the SOFA score is not adjusted for age and therefore not suitable for children. Recently, three types of pediatric SOFA (pSOFA) scores suitable for children have been proposed $[14,15,16]$. The pSOFA score used in our study was proposed by Matics and SanchezPinto in 2017, which was more consistent with the adult SOFA score [17]. The Matics and Sanchez-Pinto's scoring method is composed of six parts, including respiratory score, coagulation score, hepatic score, cardiovascular score, neurologic score and renal score. But its prognostic accuracy for in-hospital mortality still needs to be validated. Furthermore, the scoring system involves a large number of test indicators, and the scoring process is relatively complex and expensive. The feasibility of pSOFA in lowand middle-income developing countries has yet to be verified.

Located in the east of China, Shandong province has 16 cities and a total area of approximately 160,000 $\mathrm{km}^{2}$ and a total population of more than 100 million. Economically, it ranks in the middle across the country. In this study, we compared the performance of the PSOFA with SIRS criteria as predictors of inhospital mortality in children with infectious diseases from PICUs of three tertiary care hospitals of Shandong Province, China.

\section{Materials And Methods}

\section{Study design and population}

This retrospective cohort study was conducted in three pediatric intensive care units of three tertiary care hospitals in Shandong Province and was approved by the Institutional Review Board of each hospital (Suppl Table.1).

Using a uniform chart, we retrospectively reviewed the medical records of all children who were admitted to these three PICUs for the treatment of infection between January 2011 and December 2019. The chart reviews were conducted by medical residents at each hospital. Two senior attending doctors were responsible for the quality control of medical records, and assessed whether patients met the criteria and also checked the calculation of pSOFA and SIRS scores.

Inclusion criteria for patients were: $\otimes$ age ranging from $>28$ days to $<18$ years, $\otimes$ had a suspected or proven infection at admission to the PICU, $\triangle$ clinical data was complete. Patients with incomplete clinical data or who transferred to other hospitals were excluded (Fig.1).

The following data of patients was collected: general information (age, sex, and body weight), primary infection site, comorbidities, and the highest and lowest cardio-respiratory, neurologic, hepatic, renal and 
haematological organ function values during the first 24 hours following PICU admission. SIRS and pSOFA scores within the first 24 hours of PICU admission were then calculated separately according to above values.

\section{Definitions and assessment scoring methods}

Infection can be suspected or proven by a positive culture test, tissue stain or polymerase chain reaction (PCR) test for the specific pathogen, or a clinical diagnosis from a clinical exam, imaging, or laboratory test [9]. The 24h pSOFA and SIRS scores were calculated using scoring algorithms (Suppl Table 2-3). Missing values were scored as zero. The baseline pSOFA score was assumed to be zero in patients not known to have previous organ dysfunction. The duration of mechanical ventilation (MV), length of PICU stay (PICU LOS) and in-hospital mortality were recorded.

\section{Statistical analysis}

Data are presented as number (percentage), mean (standard deviation) or median (interquartile range). Descriptive analyses were used to evaluate the study population. Comparison between two groups of categorical variables was checked with a Chi-squared test. Comparison between two groups of continuous variables was checked by applying either a Student's t-test or Wilcoxon-Mann-Whitney U test according to the data distribution. Distribution of continuous variables was ascertained with the ShapiroWilk normality test.

Univariate and multivariate logistic regressions were conducted to analyze the risk factors predicting inhospital mortality. After the risk factors were screened out, an area under the relative operating characteristic curve (AUROC) analysis was performed to measure the diagnostic ability of each score [18]. When the AUROC score is between 0.90 to 1 , the ability to diagnose disease is excellent; 0.80 to 0.90 ,good; 0.70 to 0.80 ,fair; 0.60 to 0.70 ,poor; and 0.50 to 0.60 ,failure.We compared the diagnostic ability of these two evaluation methods by Delong's test. Relevant sensitivity and specificity values were calculated according to the AUROC. The optimal threshold value was then obtained by identifying the value corresponding to the maximal Yuden Index (YI= sensitivity+ specificity-1).

All statistical analyses were performed with SPSS v23.0 (SPSS, Chicago, IL) except that Delong's test was performed with Medcalc software. A P value $<0.05$ was considered statistically significant.

\section{Results}

\section{Basic PICU information from three hospitals (Table 1)}

Two of the 3 PICUs in Shandong Province are general hospitals, one located in Jinan and one in Jining. The third PICU is from a women's and children's hospital in Qingdao. The mean number of beds is 20 in the units, and all units accept medical and surgical patients. A detailed description of the participating areas and PICUs are provided in Table 1. 


\section{Overall patient characteristics (Table 2, Fig.1-2)}

Between January 2011 and December 2019, 1543 patients with ages ranging from $>28$ days to $<18$ years were admitted to these three PICUs because of confirmed or suspected infection. We excluded 451 patients: 69 patients were transferred alive to other hospitals with unknown outcome and 382 had incomplete data. Finally, 1092 patients were included in our study (Fig.1). There were 669 (61.3\%) male patients with a median age of 7.0 months (interquartile range [IQR] 2.9-19.2). The respiratory system (57.2\%) was the most common site of infection, followed by bloodstream (13.5\%) and cerebral (12.1\%) infections. In-hospital mortality was $23.4 \%$ (Table 2). The in-hospital mortality rate for patients increased with increases in their 24h pSOFA score and SIRS criteria, as shown in Figure 2. (Figure 2).

\section{Comparison of Demographic and Clinical Characteristics between Survivors and Non- survivors (Table 2- 3)}

The median age, gender ratio and proportion of surgical patients did not differ between survivor and nonsurvivor groups. The proportion of primary infections being in the digestive tract and bloodstream were higher in non-survivors ( $16.5 \%$ vs $9.0 \%, p<0.01 ; 17.6 \%$ vs $12.2 \%, p<0.05$, respectively). The proportion of primary infections being in the respiratory tract was lower in non-survivors $(48.2 \% v s 60.0 \%, p<0.001)$. The median 24h-pSOFA score of non-survivors was significantly higher than those of survivors (6.0 vs 3.0, $p<0.001)$, while the SIRS criteria value of non-survivors was similar to that of survivors.

In non-survivors, the percentage of patients receiving mechanical ventilation (MV) and continuous blood purification (CBP) treatment was $94.1 \%$ and $20.0 \%$ respectively, which was significantly higher than those in survivors $(p<0.001)$. Moreover, the duration of MV was longer in non-survivors than that in survivors ( $p<0.001)$, and the PICU LOS for non-survivors was shorter than the PICU LOS for survivors $(p<0.001)$ (Table 2).

Univariate logistic regression showed that high SIRS values, $24 \mathrm{~h}$ pSOFA values, bloodstream as the main infection site, as well as the need for MV and CBP were all risk factors related to in-hospital death. Furthermore, multivariate logistic regression showed that high $24 \mathrm{~h}$ pSOFA values, bloodstream as the main infection site, and the need for MV were associated with the higher risk of in-hospital mortality (Table 3).

\section{Comparison of SIRS and pSOFADTable 4-5)}

According to AUROC, the diagnostic ability of pSOFA values for predicting in-hospital mortality (AUROC, $0.740[99 \% \mathrm{Cl}, 0.706-0.774])$ was significantly higher than that of the SIRS criteria (AUROC, 0.559 [99\% Cl, 0.519-0.600]) (Table 5). According to the maximal Yuden Index, the optimal 24h pSOFA threshold value to predict mortality was $\geq 5$ and the optimal SIRS criteria cutoff to discriminate mortality was $\geq 3$. Next we attempted to calculate the sensitivity and specificity for predicting in-hospital mortality in infected children for pSOFA and SIRS. We used 24h pSOFA scores of $\geq 2,4$ and 5 , and we used SIRS scores of $\geq 2$ and 3 . Sensitivity and specificity results were $97.6 \%$ and $78.9 \%, 79.2 \%$ and $52.0 \%, 68.2 \%$ and $30.8 \%$, 
$74.1 \%$ and $67.4,36.5 \%$ and $62.4 \%$, respectively. Therefore, the sensitivity and specificity were highest when $24 \mathrm{~h}$ pSOFA scores were $\geq 2$ (Table 4).

In addition, we compared the cost and time required to complete filling all items in the 24h pSOFA and SIRS rating system. The average cost of completing all items in the $24 \mathrm{~h}$ pSOFA rating scale is significantly higher than that of the SIRS rating scale (307 RMB vs $37 \mathrm{RMB}, \mathrm{P}<0.001$ ). Moreover, the time required to complete the 24h-pSOFA rating form is significantly longer than the SIRS rating ( $154 \mathrm{~s}$ vs $27 \mathrm{~s}$, $\mathrm{P}<0.001$ ) (Table 4).

\section{Comparison of Simple-pSOFA with SIRS and pSOFA (Table 5-70Fig. 3)}

The pSOFA score is calculated through the assessment of six key organ functions: respiratory, coagulation, hepatic, cardiovascular, neurological, and renal. On the premise of comparison with the SIRS standard, we separately calculated the predictive values of each pSOFA sub-component (Table 6 ). The negative predictive value for a neurologic score of $\geq 1$ reached $94.23 \%$, while the positive predictive value for a hepatic score of $\geq 4$ reached $99.6 \%$. In order to better predict the prognosis of infected children, three sub-components of the pSOFA score with relatively good predictive value were screened out according to the Yuden Index. Coagulation score, cardiovascular score and the neurologic score were used to form the new scoring system, called the simple-pSOFA score (Table 7). Each indicator in the new rating system was scored on the same criteria as the pSOFA score. To verify its accuracy, the AUROC of the simplepSOFA score was calculated and compared with that of 24h-pSOFA and SIRS.

The AUROC of simple-pSOFA (AUROC, 0.751 [99\% Cl,0.716-0.786]) was significantly higher than that of SIRS (AUROC, $0.559[99 \% \mathrm{Cl}, 0.516-0.600])(\mathrm{P}=0.004)$ and pSOFA (AUROC, $0.740[99 \% \mathrm{Cl}, 0.706-0.774])(\mathrm{P}$ $<0.001$ ) (Table 5, Fig. 3). The sensitivity, specificity, negative predictive value (NPV), and positive predictive value (PPV) of simple-pSOFA were calculated for several in-hospital mortality threshold values, and a threshold value of 3 gave the best results of $65.90 \%, 74.60 \%, 87.76 \%$ and $44.09 \%$, respectively, for the four parameters (Table 5).

Although simple-pSOFA has the largest AUROC, its sensitivity and specificity for predicting in-hospital mortality of infected children are less than the $24 \mathrm{~h}$ pSOFA with a threshold value $\geq 2(97.6 \%$ and $78.9 \%)$.

\section{Discussion}

The present study aimed to compare the performance of the pSOFA scoring with the SIRS criteria as predictors of in-hospital mortality in children with infectious diseases and evaluate whether or not it is feasible to adopt pSOFA scores for use in PICUs in China. Compared with the SIRS criteria, the 24h pSOFA score showed superior prognostic accuracy in this multi center cohort study for predicting in-hospital mortality. In these 1092 children, the lung was the most common site of infection, followed by bloodstream and cerebral infection. In-hospital mortality totaled 23.4\%. Multivariable logistic regression indicated a high 24h pSOFA value, MV usage, and bloodstream as the main infection site were factors associated with the higher risk of in-hospital mortality. 
Although the paradigm of SIRS as a predictive marker of pediatric sepsis has spanned 2 decades, pediatric Sepsis-2 definitions focus on systemic inflammation $[19,20]$ but not organ dysfunction. Previous studies reported SIRS criteria being met in $>90 \%$ of febrile children present in emergency departments, of whom $<5 \%$ required ICU admission [21]. The common accepted notion is that SIRS has excellent sensitivity, but poor specificity for sepsis. Kaukonen and colleagues have previously shown that SIRS criteria is an imperfect predictor of ICU mortality, inappropriately excluding a subset of patients with infection, organ failure, and increased mortality [11]. In this study, AUROC curve analysis showed that the sensitivity and specificity of SIRS when predicti ng in-hospital mortality of infected children were $74.1 \%$ and $67.4 \%$ respectively.

In a recent study of children with bloodstream infections, SIRS criteria calculated the 30-day mortality to be $1 \%$ when calculated using the bacteraemia parameter, however this did not consider organ dysfunction. Mortality increased to $17 \%$ when organ dysfunction was included. It is important to differentiate between the definitions of "infection" versus "sepsis", the latter is defined as an infection associated with organ dysfunction [22]. SOFA scoring has been demonstrated to be a useful predictor of ICU mortality [23]. Sepsis 3.0 defined an automatic increase in SOFA scoring by $\geq 2$ points based on the need to identify the presence of emerging organ dysfunctions, to better characterize sepsis.

The pSOFA score in this study adopted the Matics and Sanchez-Pinto scoring method. The AUROC analysis verified that $24 \mathrm{~h}$ pSOFA scores were a superior mortality prediction tool relative to SIRS. A $24 \mathrm{~h}$ pSOFA score of $\geq 2$ displayed the best sensitivity and specificity for predicting in-hospital mortality (97.6\% and $78.9 \%)$.

According to the Yuden Index, the optimal 24h pSOFA threshold value to discriminate mortality was $\geq 5$. The optimal 24h pSOFA score in our study differs from that found by Matics and colleagues, which was a pSOFA of $>8$ in critically ill children [14]. Their pSOFA calculation gave the maximum pSOFA score during the whole hospital stay. The authors found that the maximum pSOFA score had excellent diagnostic ability and seemed better than the 24h pSOFA score for in-hospital mortality prognosis. However, in China, a low- and middle-income developing country, it is difficult for patients to afford the high cost required to calculate pSOFA over the whole hospital stay. So the $24 \mathrm{~h}$-pSOFA score seems more appropriate for pediatric patients in China. In this study, we calculated that the sensitivity and specificity of $24 \mathrm{~h}$ pSOFA $\geq 4$ and $\geq 5$ points were $79.2 \%$ and $52.0 \%, 68.2 \%$ and $30.8 \%$, respectively, which were not as good as that of a $24 \mathrm{~h}$ pSOFA score $\geq 2$ ( $97.6 \%$ and $78.9 \%)$.

In order to assess the feasibility of 24h pSOFA and SIRS scoring in PICUs of Shandong province, we calculated the cost and time required to complete the two rating scales respectively. It was found that the time required to complete the $24 \mathrm{~h}$-pSOFA rating form was 154 seconds, which was approximately three times longer than the 27 seconds to complete the SIRS criteria form. The cost of conducting the $24 \mathrm{~h}$ pSOFA was 607 RMB, nearly twenty times higher than the 37 RMB cost to conduct the SIRS criteria. Although 24h pSOFA score has better diagnostic ability than SIRS criteria in assessing mortality in our study, SIRS criteria is obviously implemented much more in emergency care and in developing countries 
such as China, due to its short time requirements and low cost to complete. The ideal scoring method should have the advantages of both a convenient and rapid process, high sensitivity and specificity, and low medical cost. For this we tried to combine the two scoring methods to form a new scoring method, called the simple pSOFA, in order to make full use of advantages and avoid disadvantages of the two scoring methods and find a better method to predict mortality risk.

Simple pSOFA scoring includes three sub-components, and only three indicators. Namely the Glasgow score, mean arterial pressure (MAP) and platelet count (PLT), need to be tested. The simple pSOFA score does not increase the number of required detection items, and the cost is approximately the same as the SIRS criteria and significantly lower than pSOFA calculation cost. Of the three scoring methods in this study, simple pSOFA score had the largest AUROC, suggesting that it had the best diagnostic ability in assessing in-hospital mortality of infected children. Although the sensitivity and specificity of a simple pSOFA score of $\geq 3$ is inferior in predicting in-hospital mortality of infected children when compared to a $24 \mathrm{~h}$ pSOFA score $\geq 2$, it significantly reduces the medical expenditure of the patients while providing adequate predictions of in-hospital mortality risk, and simplifies the evaluation process. The present study proposes that the SIRS criteria combined with simple-pSOFA score can be used to predict the death risk in emergency or primary hospitals, with the goal to help emergency or primary hospitals to quickly identify infected children with high risk of in-hospital death without increasing the financial burden of patients.

There are several limitations in our study. First, our results were generated using retrospective data from 3 PICUs, and the non-PICU population was not examined. The validity of the pSOFA score or similar approaches must be confirmed in other settings and populations. Second, data for analysis were collected only during the first 24 hours after PICU admission. SIRS criteria and pSOFA scores require highest and lowest recorded values, which in this study could only come from any time within the first $24 \mathrm{~h}$ of admission, and are not necessarily accurately linked to the diagnosis timing of the infection. Third, this study was limited by its retrospective design, which was susceptible to incomplete data and recall bias. Finally, due to the limited number of cases, especially in the screening of simple-pSOFA score, some measured parameters had too small of a sample size to support objective conclusions. Therefore, it is necessary to conduct a larger sample study to further verify our results.

\section{Conclusions}

Our study suggested that PSOFA had greater prognostic accuracy for in-hospital mortality in children with comfirmed or suspected infection than SIRS criteria. However, complex, time-consuming and relatively high costs limit the application of pSOFA in emergency and low- and middle-income areas. Since the majority of sepsis deaths occur in low- and middle-income developing countries in the world [24], efforts to develop a parallel pediatric definition must take into account many issues specific to low- and middleincome countries. Simple-pSOFA is proposed as a new idea to accomplish this purpose.

\section{Abbreviations}


SOFA: Sequential Organ Failure Assessment; pSOFA: Pediatric SOFA; PICU: pediatric intensive care units; AUROC: receiver operating characteristic curve; MV: mechanical ventilation; SIRS criteria:; PCR: polymerase chain reaction; PICU LOS: length of PICU stay; CBP: continuous blood purification; MAP: mean arterial pressure; PLT: platelet count.

\section{Declarations}

\section{Acknowledgements}

Not applicable.

\section{Authors' contributions}

Youpeng $\mathrm{J}$ and Chun $\mathrm{Z}$ designed the study and had full access to all data in the study. All authors of the manuscript are accountable for all aspects of the accuracy and integrity of the manuscript. Data collection: all authors; statistical analysis: Youpeng J, Jinfang Z and Yujuan W; interpretation of the data: all authors; drafting of the manuscript: Chun Z, Meiyun Xin and Jing Li; critical revision of the manuscript for important intellectual content: all authors; Final approval of the manuscript: all authors. All authors reviewed and approved the final version of the manuscript.

\section{Funding}

No funding.

\section{Availability of data and materials}

The datasets used and/or analyzed during the current study are available from the corresponding author on reasonable request.

\section{Ethical approval}

The study was approved by the medical research ethics review board of each hospital.

\section{Consent for publication}

Not applicable.

\section{Competing interests}

All authors declare that they have no conflicts of interest with the contents of this article.

\section{Author details}

${ }^{1}$ Department of Pediatric intensive care unit, Shandong Provincial Hospital Affiliated to Shandong First Medical University, Jinan, Shandong Province, P.R China, 250021. ${ }^{2}$ Department of Pediatrics, Affiliated 
Hospital of Jining Medical University, Jining, Shandong Province, P.R China, 272000. ${ }^{3}$ Department of Pediatrics, Qingdao Women and Children Hospital, Qingdao, Shandong Province, P.R China, 266000;

${ }^{4}$ Department of Pediatric intensive care unit, Shandong Provincial Hospital, Cheeloo College of Medicine, Shandong University, Jinnan, Shandong Province, P.R China, 250021.

\section{References}

1. Hartman ME, Linde-Zwirble WT, Angus DC, Watson RS. Trends in the epidemiology of pediatric severe sepsis*. Pediatr Crit Care Med. 2013;14(7):686-93.

2. Balamuth F, Weiss SL, Neuman MI, et al. Pediatric severe sepsis in U.S. children's hospitals[J]. Pediatr Crit Care Med,2014, 15(9): 798805.

3. Liu L, Johnson HL, Cousens S, et al. Global, regional, andnational causes of child mortality: an updated systematic analysis for 2010 with time trends since 2000 [published correction appears in Lancet. 2012 Oct 13;380(9850):1308]. Lancet,2012, 379(9832): 21512161.

4. Schlapbach LJ, Straney L, Alexander J, et al. Mortality related to invasive infections, sepsis, and septic shock in critically ill children in Australia and New Zealand, 2002-13: a multicentre retrospective cohort study. Lancet Infect Dis. 2015;15(1):46-54.

5. Weiss SL, Balamuth F, Hensley J, et al. The epidemiology of hospital death following pediatric severe sepsis: when, why, andhow children with sepsis die[J]. Pediatr Crit Care Med,2017,18(9):823830.

6. Kissoon N, Carcillo JA, Espinosa V, et al. World federation of pediatric intensive care and critical care societies: globalsepsis initiative[J]. Pediatr Crit Care Med. 2011;12(5):494503.

7. Boeddha NP, Schlapbach LJ, Driessen GJ, et al. Mortality and morbidity in communityacquired sepsis in European pediatric intensive care units: a prospective cohort study from the European Childhood Lifethreatening Infectious Disease Study(EUCLIDS) [J]. Crit Care. 2018;22(1):143.

8. Weiss SL, Fitzgerald JC, Pappachan J, et al. Global epidemiology of pediatric severe sepsis: the sepsis prevalence, outcomes, and therapies study [published correction appears in Am J Respir Crit Care Med. 2016 Jan 15;193(2):223-4]. Am J Respir Crit Care Med. 2015;191(10):1147-1157.

9. Goldstein B, Giroir B, Randolph A. International Consensus Conference on Pediatric Sepsis. International pediatric sepsis consensus conference: definitions for sepsis and organ dysfunction in pediatrics. Pediatr Crit Care Med. 2005;6(1):2-8.

10. Dellinger RP, Levy MM, Rhodes A, et al. Surviving Sepsis Campaign: international guidelines for management of severe sepsis and septic shock.2012. Intensive Care Med. 2013;39(2):165-228.

11. Kaukonen KM, Bailey M, Pilcher D, Cooper DJ, Bellomo R. Systemic inflammatory response syndrome criteria in defining severe sepsis. N Engl J Med. 2015;372(17):1629-38.

12. Raith EP, Udy AA, Bailey M, et al. Prognostic Accuracy of the SOFA Score, SIRS Criteria, and qSOFA Score for In-Hospital Mortality Among Adults With Suspected Infection Admitted to the Intensive Care Unit. JAMA. 2017;317(3):290-300. 
13. Singer M, Deutschman CS, Seymour CW, et al. The Third International Consensus Definitions for Sepsis and Septic Shock (Sepsis-3). JAMA. 2016;315(8):801-10.

14. Matics TJ, Sanchez-Pinto LN. Adaptation and Validation of a Pediatric Sequential Organ Failure Assessment Score and Evaluation of the Sepsis-3 Definitions in Critically III Children. JAMA Pediatr. 2017;171(10):e172352.

15. Schlapbach LJ, Straney L, Bellomo R, MacLaren G, Pilcher D. Prognostic accuracy of age-adapted SOFA, SIRS, PELOD-2, and qSOFA for in-hospital mortality among children with suspected infection admitted to the intensive care unit. Intensive Care Med. 2018;44(2):179-88.

16. Shime N, Kawasaki T, Nakagawa S. Proposal of a New Pediatric Sequential Organ Failure Assessment Score for Possible Validation. Pediatr Crit Care Med. 2017;18(1):98-9.

17. Kawasaki T, Shime N, Straney L, et al. Paediatric sequential organ failure assessment score (pSOFA): a plea for the world-wide collaboration for consensus. Intensive Care Med. 2018;44(6):995-7.

18. Hanley JA, McNeil BJ. The meaning and use of the area under a receiver operating characteristic (ROC) curve. Radiology. 1982;143(1):29-36.

19. Vincent JL, Mira JP, Antonelli M. Sepsis: older and newer concepts. Lancet Respir Med. 2016;4(3):237-40.

20. Vincent JL, Opal SM, Marshall JC, Tracey KJ. Sepsis definitions: time for change. Lancet. 2013;381(9868):774-5.

21. Scott HF, Deakyne SJ, Woods JM, Bajaj L. The prevalence and diagnostic utility of systemic inflammatory response syndrome vital signs in a pediatric emergency department. Acad Emerg Med. 2015;22(4):381-9.

22. Agyeman PKA, Schlapbach LJ, Giannoni E, et al. Epidemiology of blood culture-proven bacterial sepsis in children in Switzerland: a population-based cohort study. Lancet Child Adolesc Health. 2017;1(2):124-33.

23. Vincent JL, Moreno R, Takala J, et al. The SOFA (Sepsis-related Organ Failure Assessment) score to describe organ dysfunction/failure. On behalf of the Working Group on Sepsis-Related Problems of the European Society of Intensive Care Medicine. Intensive Care Med. 1996;22(7):707-10.

24. Kissoon N, Carapetis J. Pediatric sepsis in the developing world[J]. J Infect. 2015;71(Suppl 1):2126.

\section{Tables}

\begin{tabular}{ccccc}
\multicolumn{5}{c}{ Table 1. Description of participating pediatric intensive care units (PICUs) } \\
\hline City & Hospital & No. of beds & No. admitted & Cases of death (n) \\
& & & & \\
\hline Ji nan & General hospital & 20 & 764 & 171 \\
Qing dao & Women and children's hospital & 20 & 218 & 55 \\
Ji ning & General hospital & 18 & 110 & 29 \\
\hline
\end{tabular}


Table 2. Demographic and Clinical Characteristics of Survivors and Non-survivors with Infectious Disease

\begin{tabular}{|c|c|c|c|c|}
\hline Characteristics & $\begin{array}{c}\text { All } \\
(\mathrm{n}=1092)\end{array}$ & $\begin{array}{c}\text { Survivors } \\
(\mathrm{n}=837)\end{array}$ & $\begin{array}{c}\text { Non- } \\
\text { survivors }(\mathrm{n}=255)\end{array}$ & $\begin{array}{cl}\mathrm{P} \\
\text { value }\end{array}$ \\
\hline \multicolumn{5}{|l|}{ Clinical Variables } \\
\hline Age, months, median (IQR) & $\begin{array}{l}7.0(2.9- \\
19.2)\end{array}$ & $\begin{array}{l}7.0(2.9- \\
19.0)\end{array}$ & $6.0(2.8-24.0)$ & 0.10 \\
\hline Male, no. (\%) & $669(61.3)$ & $156(61.2)$ & $513(61.3)$ & 1.00 \\
\hline Type of admission, no. (\%) & & & & 0.45 \\
\hline Medical condition & $953(87.2)$ & $734(87.7)$ & $219(85.9)$ & - \\
\hline Surgical condition & $139(12.8)$ & $103(12.3)$ & $36(14.1)$ & - \\
\hline \multicolumn{5}{|l|}{ Admission type, n (\%) } \\
\hline Respiratory failure & 403 & $339(40.5)$ & $64(25.1)$ & $<0.001$ \\
\hline $\begin{array}{l}\text { Neurologic compromise } \\
\end{array}$ & 175 & $147(17.6)$ & $28(11.0)$ & 0.01 \\
\hline Cardiovascular compromise & 168 & $92(11.0)$ & $76(29.8)$ & $<0.001$ \\
\hline Hematologic derangement & 113 & $86(10.3)$ & $27(10.6)$ & 0.89 \\
\hline Metabolic derangement & 88 & $78(9.3)$ & $10(3.9)$ & 0.01 \\
\hline Trauma & 107 & $75(9.0)$ & $32(12.5)$ & 0.09 \\
\hline Other & 38 & $20(2.4)$ & $18(7.1)$ & $<0.001$ \\
\hline \multicolumn{5}{|l|}{ Infection source, no. (\%) } \\
\hline Bloodstream & 147 & $102(12.2)$ & $45(17.6)$ & 0.03 \\
\hline Respiratory tract & 625 & $502(60.0)$ & $123(48.2)$ & 0.001 \\
\hline Cerebral & 132 & $108(12.9)$ & $24(9.4)$ & 0.13 \\
\hline Genitourinary tract & 9 & $9(1.1)$ & $0(0)$ & 0.10 \\
\hline Digestive tract & 117 & $75(9.0)$ & $42(16.5)$ & 0.001 \\
\hline Other & 62 & $41(4.9)$ & $21(8.2)$ & 0.044 \\
\hline $\begin{array}{l}\text { Number of SIRS criteria, median (IQR) } \\
(\mathrm{n}=1092)\end{array}$ & $2(1-3)$ & $2(1-3)$ & $2(1-3)$ & 0.018 \\
\hline 24-hr pSOFA, median (IQR) $(\mathrm{n}=898)$ & $\begin{array}{l}4.0(2.0- \\
6.0) \\
\end{array}$ & $\begin{array}{l}3.0(2.0- \\
5.0) \\
\end{array}$ & $6.0(4.0-9.0)$ & $<0.0001$ \\
\hline \multicolumn{5}{|l|}{ Treatments } \\
\hline Mechanical ventilation, no. (\%) & 825 & $585(69.9)$ & $240(94.1)$ & $<0.001$ \\
\hline Continuous blood purification, no. (\%) & 144 & $93(11.1)$ & $51(20.0)$ & $<0.001$ \\
\hline \multicolumn{5}{|l|}{ Outcomes } \\
\hline Ventilator days, median (IQR) & & $4(0-9)$ & $6(1-12)$ & $<0.001$ \\
\hline PICU LOS, d, median (IQR) & & $14(8-24)$ & $10(3-16)$ & $<0.001$ \\
\hline
\end{tabular}

Abbreviations: IQR, interquartile range; LOS, length of stay;

Table 3 Univariable and multivariable logistic regression to analyze the risk factors of inhospital mortality 


\begin{tabular}{|c|c|c|c|c|c|c|c|c|c|c|c|}
\hline & \multicolumn{4}{|c|}{ Univariable logistic regression } & & & \multicolumn{5}{|c|}{ multivariable logistic regression } \\
\hline & B & SE & Wald & $\begin{array}{l}\mathrm{P} \\
\text { value }\end{array}$ & OR $(95 \%$ & CI) & B & SE & Wald & $\begin{array}{l}\mathrm{P} \\
\text { value }\end{array}$ & $\begin{array}{l}\text { OR } \\
(95 \% \\
\text { CI })\end{array}$ \\
\hline Age & 0.054 & 0.028 & 3.625 & 0.057 & $\begin{array}{l}1.055 \\
1.115)\end{array}$ & $(0.998-$ & 0.081 & 0.039 & 4.324 & 0.038 & $\begin{array}{l}1.085 \\
(1.005- \\
1.171)\end{array}$ \\
\hline Male & 0.010 & 0.147 & 0.005 & 0.944 & $\begin{array}{l}1.010 \\
1.347)\end{array}$ & $(0.758-$ & 0.092 & 0.177 & 0.267 & 0.605 & $\begin{array}{l}1.096 \\
(0.774- \\
1.551)\end{array}$ \\
\hline Type of admission & -0.203 & 0.462 & 0.193 & 0.660 & $\begin{array}{l}0.816 \\
2.019)\end{array}$ & $(0.330-$ & -0.770 & 0.537 & 2.053 & 0.152 & $\begin{array}{l}0.463 \\
(0.162- \\
1.327)\end{array}$ \\
\hline Infection source & & & 58.292 & 0.000 & & & & & 55.701 & 0.000 & \\
\hline Respiratory tract & -1.196 & 0.585 & 4.188 & 0.041 & $\begin{array}{l}0.302 \\
0.951)\end{array}$ & $(0.096-$ & -0.561 & 0.785 & 0.510 & 0.475 & $\begin{array}{l}0.571 \\
(0.123- \\
2.660)\end{array}$ \\
\hline Respiratory tract(VAP) & -21.203 & 23205.422 & 0.000 & 0.999 & 0.000 & & -20.753 & 23205.422 & 0.000 & 0.999 & 0.000 \\
\hline Bloodstream & -3.784 & 0.821 & 21.239 & 0.000 & $\begin{array}{l}0.023 \\
0.114)\end{array}$ & $(0.005-$ & -2.853 & 0.979 & 8.500 & 0.004 & $\begin{array}{l}0.058 \\
(0.008- \\
0.393)\end{array}$ \\
\hline $\begin{array}{l}\text { Bloodstream } \llbracket \text { Catheter } \\
\text { related } \square\end{array}$ & -21.203 & 10377.780 & 0.000 & 0.998 & 0.000 & & -20.039 & 9708.292 & 0.000 & 0.998 & 0.000 \\
\hline Digestive tract & -1.099 & 0.882 & 1.552 & 0.213 & $\begin{array}{l}0.333 \\
1.877)\end{array}$ & $(0.059-$ & -.906 & 1.041 & 0.757 & 0.384 & $\begin{array}{l}0.404 \\
(0.052- \\
3.111)\end{array}$ \\
\hline Hepatobiliary system & -1.792 & 0.850 & 4.445 & 0.035 & $\begin{array}{l}0.167 \\
0.882)\end{array}$ & $(0.032-$ & -0.970 & 1.012 & 0.918 & 0.338 & $\begin{array}{l}0.379 \\
(0.052- \\
2.757)\end{array}$ \\
\hline pancreas & -0.288 & 0.631 & 0.208 & 0.648 & $\begin{array}{l}0.750 \\
2.583)\end{array}$ & $(0.218-$ & 1.101 & 0.848 & 1.684 & 0.194 & $\begin{array}{l}3.008 \\
(0.570- \\
15.865)\end{array}$ \\
\hline Abdominal & 0.405 & 0.782 & 0.269 & 0.604 & $\begin{array}{l}1.500 \\
6.942)\end{array}$ & $(0.324-$ & 1.692 & 1.002 & 2.853 & 0.091 & $\begin{array}{l}5.428 \\
(0.762- \\
38.649)\end{array}$ \\
\hline Genitourinary tract & 0.000 & 1.000 & 0.000 & 1.000 & $\begin{array}{l}1.000 \\
7.099)\end{array}$ & $(0.141-$ & 0.559 & 1.609 & 0.121 & 0.728 & $\begin{array}{l}1.749 \\
(0.075- \\
40.987)\end{array}$ \\
\hline Skin and soft tissue & -21.203 & 16408.711 & 0.000 & 0.999 & 0.000 & & -19.952 & 16070.201 & 0.000 & 0.999 & 0.000 \\
\hline Cerebral & -0.981 & 0.697 & 1.979 & 0.159 & $\begin{array}{l}0.375 \\
1.471)\end{array}$ & $(0.096-$ & -0.161 & 0.897 & 0.032 & 0.857 & $\begin{array}{l}0.851 \\
(0.147- \\
4.938)\end{array}$ \\
\hline joint & -0.118 & 0.642 & 0.034 & 0.854 & $\begin{array}{l}0.889 \\
3.128)\end{array}$ & $(0.253-$ & .397 & 0.835 & 0.226 & 0.634 & $\begin{array}{l}1.487 \\
(0.290- \\
7.641)\end{array}$ \\
\hline Other & -21.203 & 23205.422 & 0.000 & 0.999 & 0.000 & & -21.222 & 23205.422 & 0.000 & 0.999 & 0.000 \\
\hline not clear & -21.203 & 16408.711 & 0.000 & 0.999 & 0.000 & & -19.057 & 16048.438 & 0.000 & 0.999 & 0.000 \\
\hline SIRS & 0.202 & 0.069 & 8.426 & 0.004 & $\begin{array}{l}1.223 \\
1.402)\end{array}$ & (1.068- & -0.149 & 0.099 & 2.284 & 0.131 & $\begin{array}{l}0.861 \\
(0.709- \\
1.045)\end{array}$ \\
\hline 24-hr pSOFA & 0.284 & 0.025 & 126.857 & 0.000 & $\begin{array}{l}1.329 \\
1.396)\end{array}$ & (1.265- & 0.241 & 0.030 & 64.029 & 0.000 & $\begin{array}{l}1.273 \\
(1.200- \\
1.350)\end{array}$ \\
\hline MV & 1.964 & 0.276 & 50.477 & 0.000 & $\begin{array}{l}7.130 \\
12.257)\end{array}$ & (4.147- & 1.732 & 0.331 & 27.329 & 0.000 & $\begin{array}{l}5.653 \\
(2.953- \\
10.822)\end{array}$ \\
\hline CBP & 0.685 & 0.191 & 12.816 & 0.000 & $\begin{array}{l}1.984 \\
2.887)\end{array}$ & (1.363- & -0.176 & 0.245 & 0.581 & 0.472 & $\begin{array}{l}0.839 \\
(0.519- \\
1.354) \\
\end{array}$ \\
\hline
\end{tabular}

\section{Table}

Table 4. Time and cost required to complete SIRS and pSOFA scores 


\begin{tabular}{lcc}
\hline & Time (S $\square$ & Cost $\square$ RMB $\square$ \\
\hline SIRS & $27(24-36)$ & $37(19-49)$ \\
24h-pSOFA & $154(111.5-190.25)$ & $607(573-698)$ \\
$P$ value & $<0.001$ & $<0.001$ \\
\hline
\end{tabular}

Table 5.The predictive value of SIRS, pSOFA and simple-pSOFA for in-hospital mortality of infected children

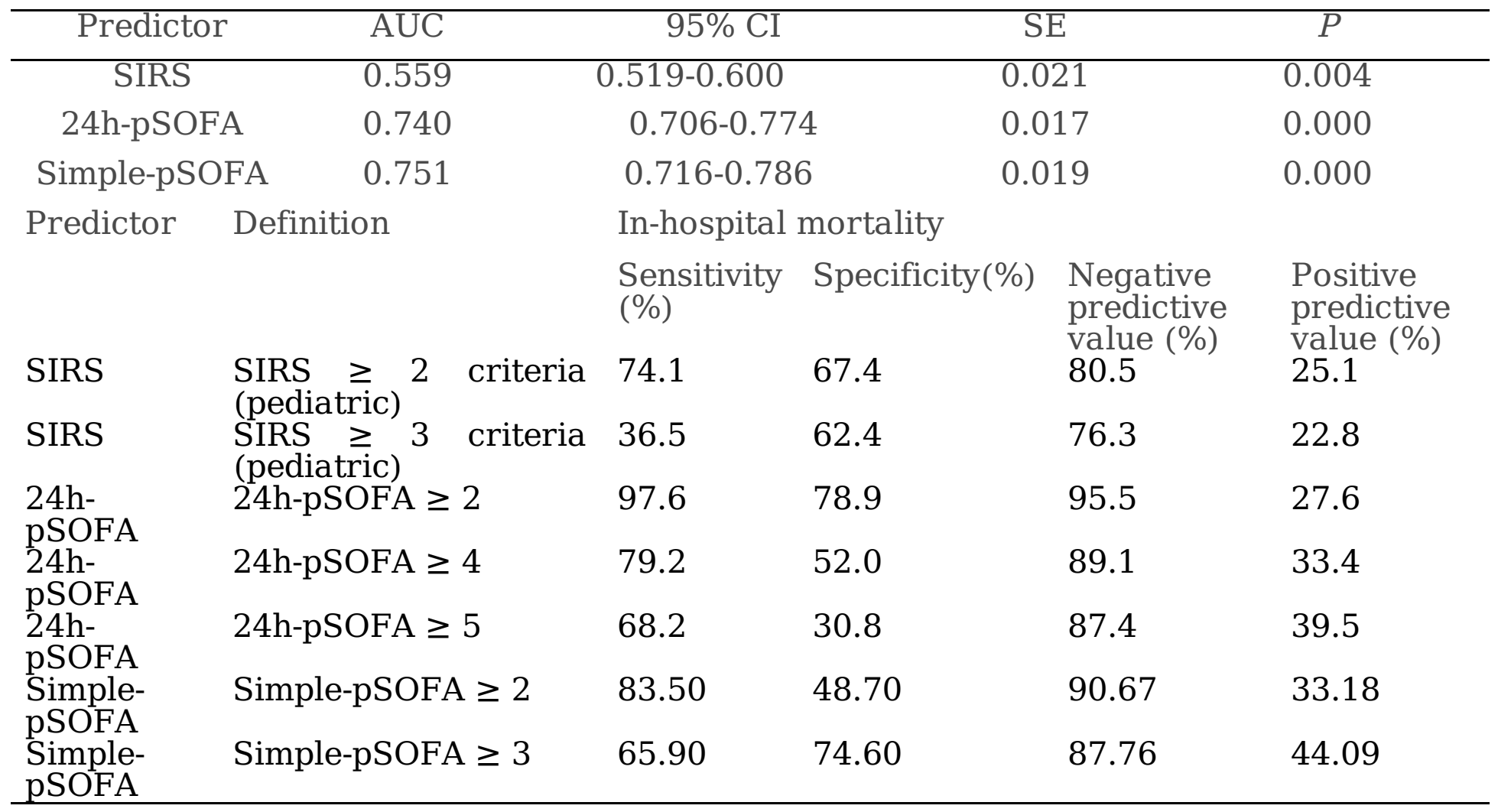

Table 6. The predictive value of different indicators in pSOFA score for in-hospital mortality of infected children 


\begin{tabular}{|c|c|c|c|c|c|}
\hline \multicolumn{6}{|c|}{ Coagulation score } \\
\hline$\geq 1$ & 126 & 25.90 & 92.80 & 80.43 & 52.38 \\
\hline$\geq 2$ & 84 & 20.00 & 96.10 & 79.76 & 60.71 \\
\hline$\geq 3$ & 39 & 8.20 & 97.80 & 77.78 & 53.85 \\
\hline$\geq 4$ & 18 & 3.50 & 98.90 & 77.09 & 50.00 \\
\hline \multicolumn{6}{|c|}{ Respiratory score } \\
\hline$\geq 1$ & 747 & 74.10 & 33.30 & 80.87 & 25.30 \\
\hline$\geq 2$ & 516 & 52.90 & 54.50 & 79.17 & 26.16 \\
\hline$\geq 3$ & 249 & 34.10 & 80.60 & 80.07 & 34.94 \\
\hline$\geq 4$ & 60 & 12.90 & 96.80 & 78.49 & 55.00 \\
\hline \multicolumn{6}{|c|}{ Cardiovascular score } \\
\hline$\geq 1$ & 186 & 34.10 & 88.20 & 81.46 & 46.77 \\
\hline$\geq 2$ & 132 & 28.20 & 92.80 & 80.94 & 54.55 \\
\hline$\geq 3$ & 93 & 21.20 & 95.30 & 79.88 & 58.06 \\
\hline$\geq 4$ & 18 & 5.90 & 99.60 & 77.65 & 83.33 \\
\hline \multicolumn{6}{|c|}{ Neurologic score } \\
\hline$\geq 1$ & 936 & 96.50 & 17.60 & 94.23 & 26.28 \\
\hline$\geq 2$ & 570 & 74.10 & 54.50 & 87.36 & 33.16 \\
\hline$\geq 3$ & 270 & 47.10 & 82.10 & 83.58 & 44.44 \\
\hline$\geq 4$ & 69 & 20.00 & 97.80 & 80.06 & 73.91 \\
\hline \multicolumn{6}{|c|}{ Hepatic score } \\
\hline$\geq 1$ & 144 & 18.80 & 88.50 & 78.16 & 33.33 \\
\hline$\geq 2$ & 87 & 14.10 & 93.90 & 78.21 & 41.38 \\
\hline$\geq 3$ & 27 & 7.10 & 98.90 & 77.75 & 66.67 \\
\hline $\begin{array}{l}\geq 4 \\
\text { Rena }\end{array}$ & 9 & 3.50 & 99.60 & 77.29 & 99.60 \\
\hline$\geq 1$ & 186 & 29.40 & 86.70 & 80.13 & 40.32 \\
\hline$\geq 2$ & 96 & 16.50 & 93.50 & 78.61 & 43.75 \\
\hline$\geq 3$ & 63 & 9.40 & 95.30 & 77.55 & 38.10 \\
\hline$\geq 4$ & 30 & 2.40 & 97.10 & 76.55 & 20.00 \\
\hline
\end{tabular}

Table 7 Simple-Pediatric Sequential Organ Failure Assessment Score 


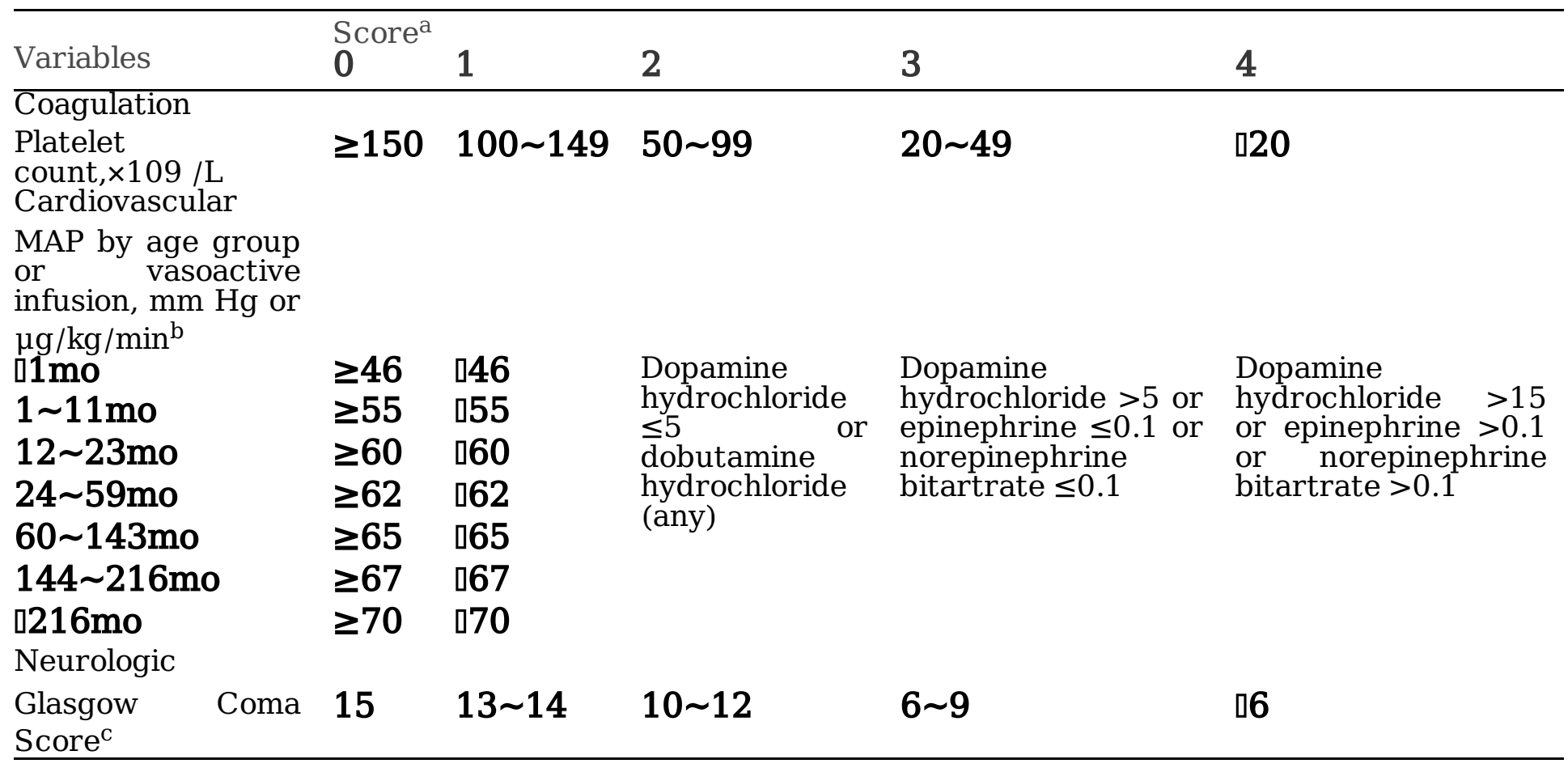

Abbreviations: MAP, mean arterial pressure; Simple-pSOFA, Simple-Pediatric Sequential Organ Failure Assessment Score.

a The Simple-pSOFA score was calculated for every 24 -hour period. The worst value for every variable in each 24-hour period was used to calculate the subscore for each of the 3 organ systems. If a variable was not recorded in a given 24-hour period, it was assumed to be normal and a score of 0 was used. Daily simple-pSOFA score was the sum of the 3 subscores (range, 0-12 points; higher scores indicate a worse outcome).

b MAP (measured in millimeters of mercury) was used for scores 0 and 1; vasoactive infusion (measured in micrograms per kiligram per minute), for scores 2 to 4 . Maximum continuous vasoactive infusion was administered for at least 1 hour.

${ }^{\mathrm{c}}$ Glasgow Coma Scale was calculated using the pediatric scale.

\section{Figures}




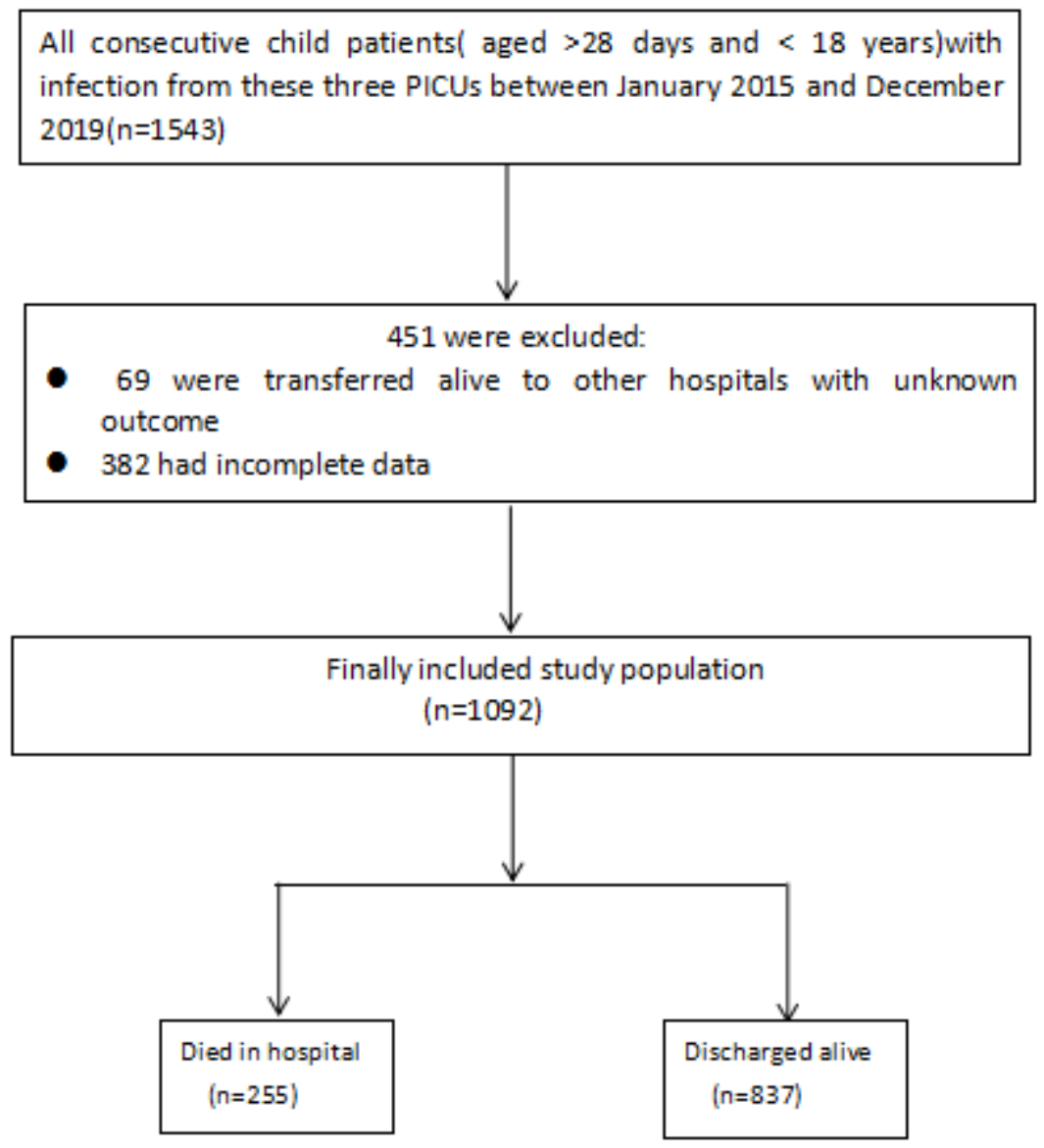

\section{Figure 1}

Flowchart of selection of patients with infection

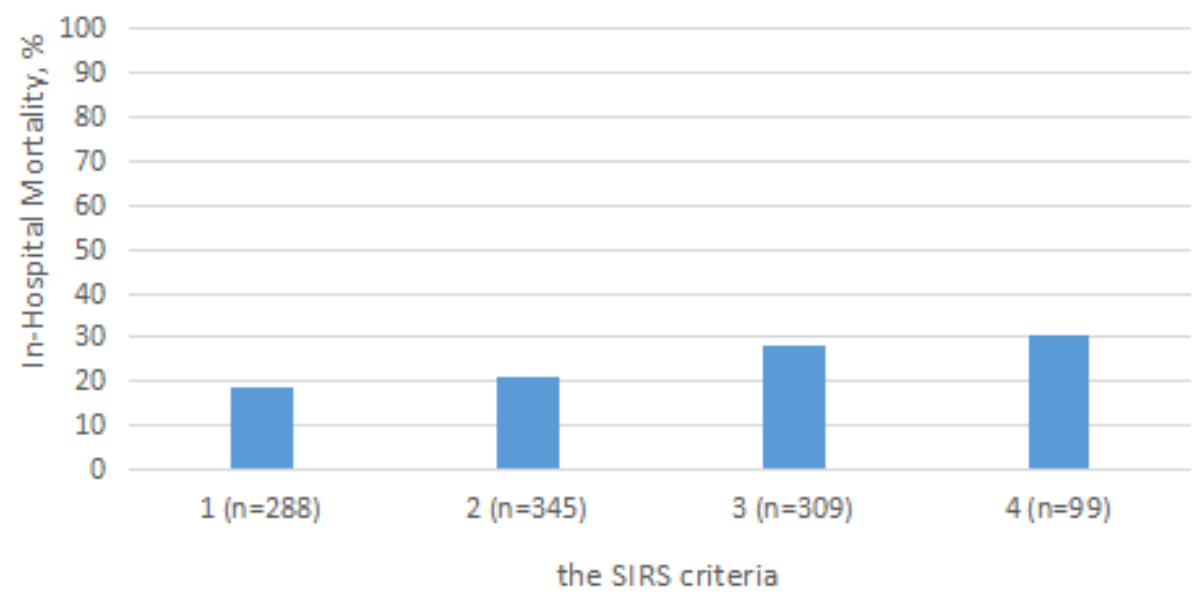

Figure 2

In-Hospital Mortality Rate Based on the SIRS criteria. The SIRS criteria is the score given within 24 hours of admission in the pediatric intensive care unit. Error bars represent 95\% Cls. (Figure 2-1 in the manuscript) 


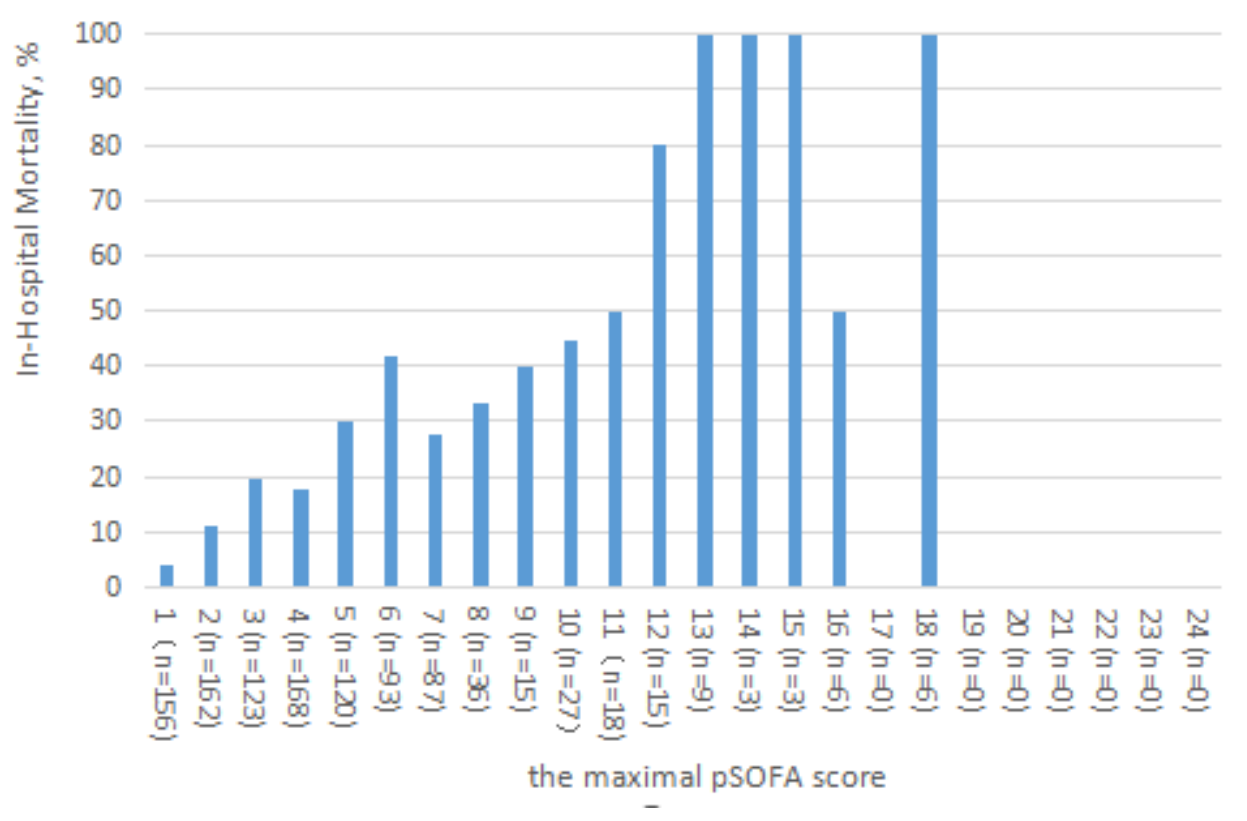

\section{Figure 3}

In-Hospital Mortality Rate Based on the maximal pSOFA Score. The maximal pSOFA Score is the score given within 24 hours of admission in the pediatric intensive care unit. Error bars represent $95 \% \mathrm{Cls}$.

(Figure 2-2 in the manuscript)
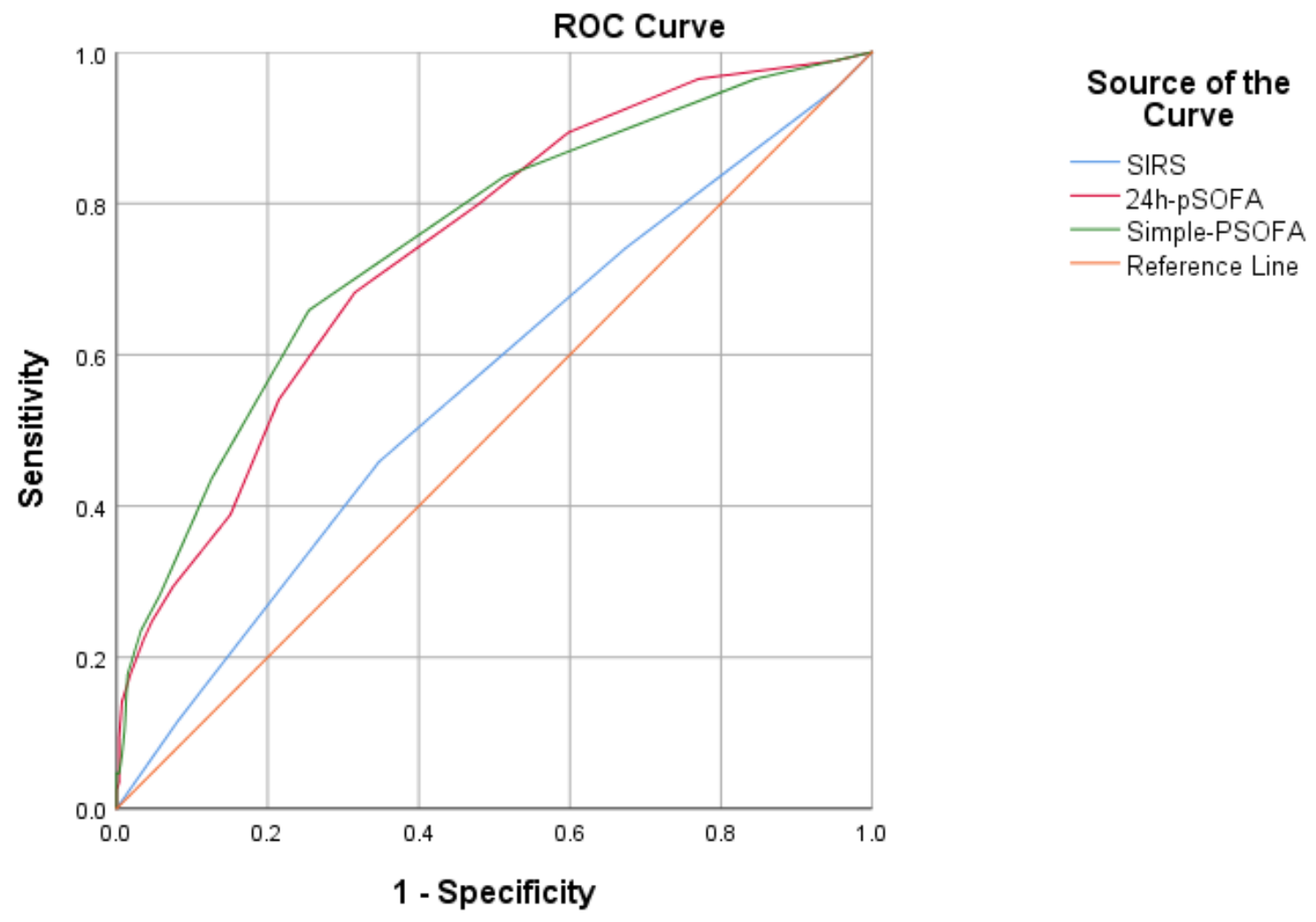

Diagonal segments are produced by ties.

Figure 4 
AUROC curve of SIRS,24h-pSOFA and Simple-pSOFA for in-hospital mortality in patients with suspected infection. Abbreviations: AUROC, area under the receiver operating characteristic; SIRS, systemic inflammatory response syndrome.24h-pSOFA, sequential (sepsis-related) organ failure assessment. (Figure 3 in the manuscript)

\section{Supplementary Files}

This is a list of supplementary files associated with this preprint. Click to download.

- suppltable.docx 